\title{
Ethanol Effects on Cortical EEG Correlation and Sexual Behavior in Male Rats
}

\author{
Miguel Angel Guevara1, Marisela Hernández-González ${ }^{1}$, Mayra Linné Almanza-Sepúlveda ${ }^{1}$, \\ Diego Radberto Chapa García Abascal ${ }^{1}$, Pilar Durán ${ }^{2}$, Carolina Sotelo Tapia ${ }^{1}$, Fabricio Luna Torres ${ }^{1}$ \\ ${ }^{1}$ Instituto de Neurociencias, Universidad de Guadalajara, Guadalajara, Jalisco, México \\ ${ }^{2}$ Departamento de Biología Celular, Facultad de Ciencias, Universidad Nacional Autónoma de México, \\ México DF, México \\ Email: mariselh@cencar.udg.mx
}

Received January 9, 2014; revised February 7, 2014; accepted February 15, 2014

Copyright (c) 2014 Miguel Angel Guevara et al. This is an open access article distributed under the Creative Commons Attribution License, which permits unrestricted use, distribution, and reproduction in any medium, provided the original work is properly cited. In accordance of the Creative Commons Attribution License all Copyrights (C) 2014 are reserved for SCIRP and the owner of the intellectual property Miguel Angel Guevara et al. All Copyright (c) 2014 are guarded by law and by SCIRP as a guardian.

\section{ABSTRACT}

Ethanol affects sexual behavior drastically, as much as its execution as well as its motivation. Ethanol is a central nervous system depressant and the prefrontal cortex (PFC) is one of the cortical areas most sensitive to its effects. This prefrontal area participates in the modulation of the cognitive control, and furthermore, by mean of connections with the parietal cortex (PC) plays an important role in the processing of sexually relevant stimuli. Considering that it has been reported that the ethanol affects the proper functioning of these cortical areas, particularly decreasing the degree of electroencephalographic (EEG) coupling or correlation between them, the aim of this study was to characterize the effect of a low dose of ethanol on EEG correlation, as well determining if this effect is associated with impaired sexual behavior in male rats. Nine male rats unilaterally implanted were simultaneously recorded the EEG from the PFC and PC to calculate the degree of EEG synchronization (correlation, $r$ ) between both cortices during the first 40 minutes post administration of $0.75 \mathrm{~g} / \mathrm{kg}$ of ethanol. At the end of the EEG recording, the males were submitted to copulatory behavior with a receptive female rat. The ethanol administration was associated with a decreased intrahemispheric correlation of the fast bands between PFC and PC, as well as with an increased mount and intromission latency. This data showed that ethanol in moderate doses decreased the coupling degree between cortices. It is likely that this functional disconnection between the PFC and PC after the ethanol administration could be associated with the difficulty in discriminating incentive value of the sexual stimuli so that decreased the sexual motivation but not the performance of the copulatory parameters in the male rat.

\section{KEYWORDS}

\section{Ethanol; Sexual Behavior; EEG; Prefrontal Cortex; Parietal Cortex; Rat}

\section{Introduction}

Sexual behavior is a motivated behavior typical of all the species, in which individuals display a range of behavior patterns in order to attract a potential sexual match and have sexual interaction [1].

This behavior is affected by the intake of different substances, most particularly by ethanol, which can significantly alter the performance of the copulatory acts as well as the structure and function of the cerebral areas, causing functional changes in both cortical and subcor- tical areas depending on the dose, route of administration and age of subjects, among others [2].

The effect of ethanol on the nervous system and its speed of action is due in part to its water solubility resulting in rapid absorption into the blood (between 30 40 minutes after) and widely distributed throughout the brain [3]. The changes occurring in regions such as the hippocampus or in the neocortex are particularly important because they are structures involved in modulating motivated behaviors, such as sexual behavior [4,5] as 
well as processes such as attention, memory and executive processes, which are affected by ethanol [6]. In fact, a high percentage of alcoholics demonstrates significant sexual $[7,8]$ and neuropsychological dysfunctions $[9,10]$ and some results with EEG recording have shown that ethanol induces important changes in the functionality of the PFC and Parietal Cortex during sexual arousal [11] and cognitive tasks [12]. In rats similar alterations occur, so that after the ethanol administration $(1 \mathrm{~g} / \mathrm{kg})$ increased, rodent sexual motivation but impaired specific parameters of sexual performance has been reported [13]; moreover, increases in the activity of dopaminergic neurons of the ventral tegmental area (VTA) that result in an increase in the levels of dopamine primarily in the shell of the nucleus accumbens (Acc) $[14,15]$ in a dose-dependent manner [16-21] have also been demonstrated. Similarly, EEG studies have shown that ethanol affects the brain activity immediately after the acute administration of a low dose, inducing a decreased theta and increased beta EEG activity in both, VTA and Acc in adult male rats $[22,23]$.

The recording of the EEG from cortical and subcortical structures has constituted a useful tool to relate changes in brain electrical activity with the performance of various behavioral responses [24,25]. The EEG, defined as a mixture of rhythmic sinusoidal-like fluctuations in voltage generated by the brain, represents the global activity of the pyramidal cells of the cortex and the activity of neurons in the subcortical structures. Thus, the quantitative analysis of EEGs has allowed researchers to investigate the simultaneous functioning of several brain structures in a precise temporal relationship with specific physiological states, behaviors and sensorial processing. One advantage of the EEG recording is that it allows the obtaining of an index of the functional coupling between two different brain sites, so that by means of the calculation of the correlation parameter it is easy to know the degree of similarity between EEG activity from two brain sites [26].

Considering that the PFC and its connections with the PC play an important role in modulating motivated behaviors, such as sexual behavior [4,5] and that this cortical areas are highly sensitive to the ethanol effects, it is most likely that ethanol changes degree of synchronization between the prefrontal and parietal cortices. Thus, the aim of this study was to characterize the acute effect of a low dose of ethanol on prefronto-parietal EEG correlation as well as on the copulatory parameters of male rats.

\section{Material and Methods}

\subsection{Subjects}

Wistar male rats, 80 - 90 days old, were obtained from a colony bred in the Institute of Neurosciences, University of Guadalajara. All males were maintained in a room at $22^{\circ} \mathrm{C}-23^{\circ} \mathrm{C}$, under a $12: 12 \mathrm{~h}$ reversed light/dark cycle (lights on from 2000 to $0800 \mathrm{~h}$ ) and housed in individual cages. Food and water were available ad libitum. Temperature, feeding, and light-dark cycle conditions were maintained constant in the course of the study. Animal care as well as all procedures involving animals was approved by our Institutional Animal Care and Use Committee, which is in accordance with the NIH specifications.

\subsection{Surgery}

For surgery, a total of 15 male rats were anaesthetized with sodium pentobarbital (35 mg/kg i.p.). Stainless steel electrodes (0.2 $\mathrm{mm}$ in diameter) were unilaterally implanted in the left hemisphere specifically into the prelimbic region of the PFC (3.2 mm anterior to bregma, 0.6 $\mathrm{mm}$ lateral to the midline, and $3.2 \mathrm{~mm}$ below the dura matter) and into the PC [ $-4.16 \mathrm{~mm}$ posterior to Bregma, and $4.5 \mathrm{~mm}$ lateral to the midline, and $1.6 \mathrm{~mm}$ below the dura matter], according to the stereotaxic atlas of Paxinos and Watson [27]. Two stainless screws were placed in the anterior and posterior parts of the skull and served as "reference" and "ground" electrodes, respectively. All electrodes were attached to a miniature connector that was fixed on the skull by means of stainless-steel hooks and acrylic cement. Adequate care was taken to minimize pain or discomfort of the animals throughout the experiment. After the surgery, all the subjects were housed in individual cages with food and water ad libitum.

\subsection{EEG Tests}

Seven days after electrode implantation, and 3 or 4 days before the EEG tests, all males were allowed to adapt to the recording room (at $22^{\circ} \mathrm{C}-23^{\circ} \mathrm{C}$ and illuminated with dim red light) and to the connection cable for $30 \mathrm{~min}$. The EEG recording was performed during the dark phase of the light-dark cycle. The day of EEG recording, was administered intraperitoneally (i.p.) a dose of $0.75 \mathrm{~g} / \mathrm{kg}$ of ethanol (99.8) or saline, both treatments were administered to each rat in a counterbalanced manner within a period of four days between each other. Immediately after the i.p administration of the ethanol or saline, the males were placed in the recording cage (clear Plexiglas cages of $43 \times 53 \times 20 \mathrm{~cm}$, with clean sawdust bedding) and were connected to the polygraph for the simultaneous recording of the EEG from the PFC and PC during $40 \mathrm{~min}$. The EEG was recorded only when the males remain in an awake-quiet state. At the end of 40 min EEG recording, the male rats were desconected, and a receptive female rat was introduced to the recording cage with the aim that the male rat performs sexual interaction until 
reach the first intromission of the second copulatory series.

\subsection{Recording of the EEG Activity}

For the recording of the EEG, the connection cable from the electrodes of the implanted rat was passed through a hole in the center of the top of a wire cage $(73 \times 57 \times 61$ $\mathrm{cm}$ ), where the plexiglas recording cage was placed. This arrangement allowed the free movement of the rat within the recording cage while the EEG from PFC and PC of the left hemisphere were continuously recorded. The cable was connected to AC preamplifiers of a Grass 7B polygraph (band pass $3-30 \mathrm{~Hz}$ ), and their outputs were plugged to a PCL-812 analog to digital converter (Advantech, Co.), which operated as an interface to a microcomputer. The EEG signals were recorded at a sampling rate of $512 \mathrm{~Hz}$ during 2 sec EEG epochs and were calibrated with a pulse of 50 microvolts $(\mu \mathrm{V})$ produced by the preamplifiers and delivered to a microcomputer as a reference to convert the output of the analog to digital converter to $\mu \mathrm{V}$. The capture of the EEG signals corresponding to each one of the two different conditions (ethanol or saline administration) was performed by means of a board unit with eight on/off buttons that were connected to digital input lines of the analog to digital converter. Thus, the computational capture of several 2 sec segments of EEG corresponding to 40 minutes after the solution administration, during the awake quiet state, started when a specific button of the board unit was pressed; another button was pushed to finish the input of signals.

By means of specific computer programs [28], the capture of the recording of the EEG signals from left PFC and PC was made in a precise temporal relation during the awake quiet state. Various EEG segments of 2 sec were captured in each condition (ethanol or saline) and were independently stored in files for off-line analysis.

\subsection{EEG Analysis}

EEG signals were carefully inspected before analysis and segments containing artifacts were discarded. At least 30 EEG segments of 2 sec from each subject during awake quiet state, in each condition (ethanol or saline) were analyzed. With the aim to obtain the degree of functional synchronization between PFC and PC of the left hemisphere, the intrahemispheric correlation was obtained in each band analyzed (4 - 7, $8-13$ and $14-30 \mathrm{~Hz}$ ) by means of Pearson product-moment correlation coefficients.

\subsection{Sexual Behavioral Testing Procedure}

Immediately after finishing the 40-minute EEG recording, mating tests were performed. The following parameters of sexual behavior were registered: Mount and intromission latency $(M L, I L)$, the time between introduction of the female and the first mount with pelvic thrusting and the first vaginal penetration, respectively; ejaculation latency $(E L)$, the time between the first vaginal penetration (intromission) and ejaculation; postejaculatory interval (PEI), the time between ejaculation and the first intromission of the following series. The number of preejaculatory mounts (NM) and intromissions (NI) was also recorded. In addition, the intromission ratio (Hit Rate HR) (number of intromissions/(number of mounts + number of intromissions) was calculated. This ratio is supposed to reflect the motor and sensory efficiency of penile mechanisms essential for the achievement to vaginal penetration [1]. The mating test was ended at the end of the first postejaculatory interval or when the mount latency was larger than 120 sec.

\subsection{Histology}

At the end of each experiment, the animals were deeply anesthetized with sodium pentobarbital. Intracardial infusion of isotonic saline $(0.9 \%)$ followed by $5.0 \%$ buffered paraformaldehyde solution fixed the brain, which was afterwards removed and stored in formalin for at least two weeks; 50 micron thick sections were made with a microtome and were stained with cresyl violet. The inspection under a stereoscopic microscope following the stereotaxic coordinates allowed the reconstruction of the path followed by the recording electrode. Only those recordings obtained from the prelimbic region of the PFC, and PC were included in the analysis of data.

\subsection{Statistics}

Statistical analysis was limited to $4-30 \mathrm{~Hz}$, since lower frequencies may include artifacts arising from cable movements. By means of the Student $t$ test for correlated groups, the correlation values of the different frequency EEG bands were compared in the two conditions (ethanol or saline). For statistical purposes, correlation values were transformed to Fisher's Z-scores. Significance was set at $p \leq 0.05$ (two-tailed). The sexual interaction parameters were also compared with the same Student $t$ test for correlated groups.

\section{Results}

The tips of the electrodes were correctly placed in 9 of the 15 male rats; the PFC electrodes were unilaterally located between 3.7 and $3.2 \mathrm{~mm}$ anterior to bregma (in the prelimbic region of the PFC), while the PC electrodes were placed between -4.16 to $-4.30 \mathrm{~mm}$ posterior to Bregma (Figure 1). 


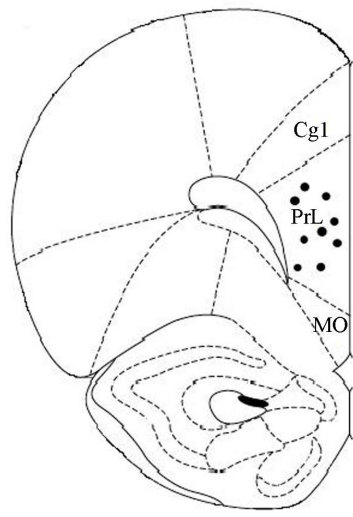

(a)

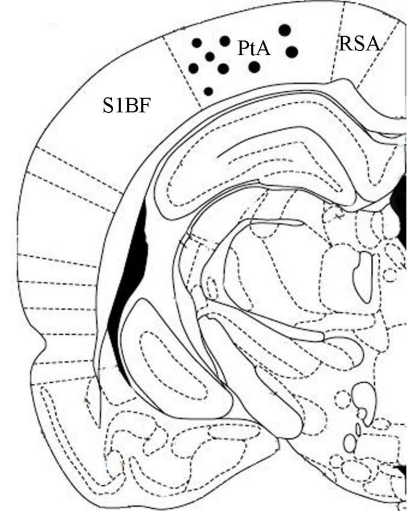

(b)
Figure 1. Schematic representation of electrode tip placements in the left hemisphere specifically into the (a) PFC (prelimbic PrL region) (3.2 mm anterior to bregma, $0.6 \mathrm{~mm}$ lateral to the midline, and $3.2 \mathrm{~mm}$ below the dura matter) and $B)$ into the PC $(-4.16 \mathrm{~mm}$ posterior to Bregma, and 4.5 $\mathrm{mm}$ lateral to the midline, and $1.6 \mathrm{~mm}$ below the dura matter), $(n=9)$, according to the stereotaxic atlas of Paxinos and Watson [27]. MO, medial orbital cortex; PrL, prelimbic cortex; Cg1, cingulated cortex area; PtA, parietal association cortex RSA, retrospenial agranular cortex; S1BF, S1 cortex barrel field. Anterior-posterior coordinates are given with respect to bregma.

\subsection{Intrahemispheric Correlation}

In general terms was found that the administration of a low doses of ethanol was associated with a decrease in the synchronization degree between PFC and PC (intrahemispheric correlation) of the three EEG frequency bands analyzed, though statistical significance only was achieved in the fast frequency band $(14-30 \mathrm{~Hz})(t=$ 2.063; $p=0.036$ ) (Figure 2).

\subsection{Sexual Behavior}

The acute i.p. administration of low doses of ethanol was associated with an increase in the mount $(t=-1.74 ; p=$ $0.042)$ and intromission $(t=-1.97 ; p=0.048)$ latencies (Table 1). All other copulatory parameters (ejaculation latency, postejaculatory interval as well as number of mounts, intromissions and hit rate) showed similar values after ethanol or saline administration.

\section{Discussion}

The results of this study showed that a moderate ethanol dose decreased the degree of synchronization between prefrontal and parietal cortices and that this lower cortical coupling was also associated with an increased mount and intromission latency, without affecting the efficiency of the male rat sexual interaction.

It is known that ethanol is a central nervous system depressant and that the prefrontal cortex is one of the cere-

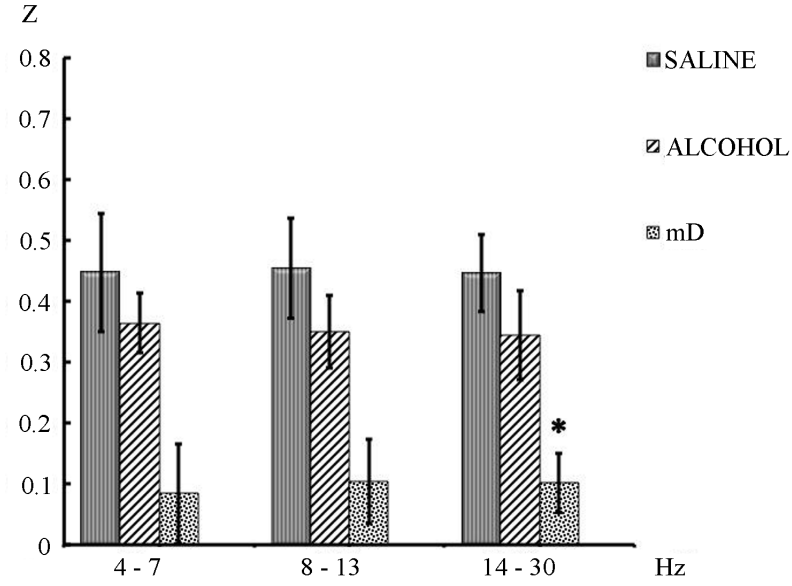

Figure 2. Mean \pm SE and mean of differences $(\mathrm{mD})$ of the intrahemispheric correlation ( $\mathrm{z}$ values) between prefrontal and parietal cortex of the three frequency bands recorded during 40 min post i.p. ethanol or saline administration in awake-quiet male rats. Student's $t$ tests for correlated groups. ${ }^{*} p \leq 0.05$ compared to saline condition.

bral areas most sensitive to its effects. Furthermore, the important role that the prefrontal cortex plays in the processing of sexually relevant stimuli (such as odor and vocalizations emitted by a receptive female rat) has been reported [29-31]. In males rats, PFC injury and exposed to a receptive female, it induces an increase in the time required to initiate intercourse, but once initiated it, the male courtship behavior is normal [29,32]. Other studies have shown the role of the prefrontal cortex in the motivational aspects of motor performance and sexual behavior $[30,32]$ and there is also evidence that this structure is involved in the processing of stimuli of potential incentives to generate sexual arousal and motivation (for a review, see [5]). In studies with humans, it has been also demonstrated that ethanol affects the functioning of the prefrontal cortex, which has been suggested that it could interfere with the adequate processing of visual erotic stimuli [11]. Thus, it is likely that in this study the ethanol could affect the proper functioning of this cortical area, decreasing the degree of coupling or synchronization with the parietal cortex. Correlation data obtained in this work are also consistent with studies in humans, which have showed that immediately after the ethanol administration, the degree of EEG coupling between prefrontal and parietal cortices decreased [33].

Schwartz and colleagues [34] have suggested that during the first 30 minutes, also called the absorption phase [ascending levels of blood ethanol concentration (BAC)], ethanol acts as a stimulant of the nervous system through processes of desinhibition. However, in the subsequent elimination phase (BAC descending levels), depressant and sedative effects are exerted. These authors also reported an increase in the alpha and beta EEG bands to- 
Table 1. Mean \pm SE of the copulatory parameters showed by male rats after the administration of ethanol or saline. Mount latency (ML), intromission latency (IL), ejaculation latency (EL) and postejaculatory interval (PEI) in seconds; number of mounts (NM), number of intromissions (NI) and hit rate (HR).

\begin{tabular}{cccccccc}
\hline & ML & IL & EL & PEI & NM & NI & HR \\
\hline Saline & $18.4 \pm 3.7$ & $18.25 \pm 3.7$ & $235.1 \pm 60.6$ & $322.3 \pm 19.8$ & $5.55 \pm 1.1$ & $7.11 \pm 0.8$ & $0.408 \pm 0.04$ \\
Ethanol & ${ }^{*} \mathbf{5 5 . 2 1} \pm \mathbf{2 1 . 3}$ & ${ }^{*} \mathbf{4 6 . 1 1} \pm \mathbf{1 4 . 6}$ & $359.0 \pm 30.01$ & $327.4 \pm 16.2$ & $8.55 \pm 4.4$ & $7.0 \pm 0.6$ & $0.404 \pm 0.07$ \\
\hline
\end{tabular}

Student's t tests for correlated groups ${ }^{*} p<0.05$ compared to saline condition.

gether with a decreased activity of delta and theta bands during the ascending phase of BAC, whereas an inverse pattern (increase in delta and theta and decrease of alpha and beta bands) was observed during the BAC downward. In a study conducted in our laboratory [23], we also found during the absorption phase an increase of the fast frequencies $(13-21 \mathrm{~Hz})$ in the Acc and VTA as well as a decreased interaccumbens correlation in the theta frequencies (4 - 7 and 8 - $12 \mathrm{~Hz}$ bands) after administration of a moderate dose of ethanol $(0.75 \mathrm{~g} / \mathrm{kg})$. An EEG pattern of increased low-voltage fast frequencies has been traditionally associated with an activated state [35], so this elevated EEG activity has been considered as an index of a state of arousal in animals and humans. Similarly, numerous studies have described irregular, low-voltage fast activity (from 12 to over $40 \mathrm{~Hz}$ ) in the cortex under various behavioral situations of increased alertness [36], or as a response to optimal sensory stimuli in animals and humans. The fact that in this study the lowest correlation was founded in the fast frequency bands may be associated with poor processing of sensory signals from the receptive female rat so that the male rats required more time to perceive it as a sexual reward, as can be determined by the increased mount and intromission latency.

Mount and intromission latency are parameters that traditionally have been considered as indicators of sexual motivation. In the condition of ethanol, male rats took longer to start intercourse than when they were treated with saline. Since the prefrontal cortex is involved in the proper processing of sexually relevant stimuli $[5,29]$, it is likely that the effects of ethanol on the prefrontal and parietal interaction could be associated with a deficient processing of the stimuli emitted by the receptive female, thus delaying the induction of the sexual motivation state. These results agree well with the data reported by Agmo et al. [29] who showed that the prefrontal cortex lesion only affects the motivational component of sexual interaction, dramatically increasing the time it took male rats to initiate intercourse, but once started, it was executed appropriately, similar to what we obtained when male rats were treated with the administration of ethanol.

It is likely that the functional disconnection between the PFC and PC after the ethanol administration could be associated with the difficulty in discriminating incentive value of the sexual stimuli so that decreased the sexual motivation but not the performance of the copulatory parameters in the male rat. These findings may contribute to improving our understanding of the brain mechanisms that underlie ethanol's effects on the processing of sexual stimuli.

\section{REFERENCES}

[1] R. L. Meisel and B. D. Sachs, "The Physiology of Male Sexual Behavior,” In: E. Knobil and J. Neill, Eds., The Physiology of Reproduction, Raven Press, New York, Vol. 2, 2nd Edition, 1994, pp. 3-96.

[2] S. Brailowsky, "Las Sustancias de los Sueños: Neuropsicofarmacología,” FCE-CONACYT, México, 1995.

[3] R. S. Feldman, J. S. Meyer and L. F. Quenzer, "Principles of Neuropsychopharmacology,” Sinauer Associates, Inc., Sunderland, 1997.

[4] T. M. Tzschentke, "The Medial Prefrontal Cortex as Part of the Brain Reward System,” Amino Acids, Vol. 19, No. 1, 2000, pp. 211-219. http://dx.doi.org/10.1007/s007260070051

[5] M. Hernández-González and M. A. Guevara, "Participation of the Prefrontal Cortex in the Processing of Sexual and Maternal Incentives," In: L. LoGrassso and G. Morretti, Eds., Prefrontal Cortex: Roles, Interventions and Traumas, Nova Science Publishers, 2009, pp. 115-157.

[6] D. Beracochea, T. P. Durkin and R. Jaffard, "On the Involvement of the Central Cholinergic System in Memory Deficits Induced by Long-Term Ethanol Consumption in Mice," Pharmacology, Biochemistry and Behavior, Vol. 24, No. 3, 1986, pp. 519-524. http://dx.doi.org/10.1016/0091-3057(86)90551-4

[7] D. B. Abrams and T. G. Wilson, "Ethanol, Sexual Arousal, and Self-Control," Journal of Personality and Social Psychology, Vol. 45, No. 1, 1983, pp. 188-198. http://dx.doi.org/10.1037/0022-3514.45.1.188

[8] G. Farkas and R. C. Rosen, "The Effects of Ethanol on Male Sexual Arousal,” Journal of Studies on Alcohol, Vol. 37, 1976, pp. 265-272.

[9] N. Landa, J. Fernández-Montalvo and J. Tirapu, “Alteraciones Neuropsicológicas en el Alcoholismo: Una Revisión Sobre la Afectación de la Memoria y las Funciones Ejecutivas,” Adicciones, Vol. 16, No. 1, 2004, pp. 41-52.

[10] L. Roehrich and M. S. Goldman, "Experience-Dependent Neuropsychological Recovery and the Treatment of Al- 
coholism," Journal of Consulting and Clinical Psychology, Vol. 61, No. 5, 1993, pp. 812-821.

http://dx.doi.org/10.1037/0022-006X.61.5.812

[11] M. Hernández-González, A. Sanz-Martin, M. A. Guevara, C. Amezcua-Gutiérrez and M. Ruiz Díaz, "Alcohol Suppresses the Electroencephalographic Response to Visual Erotic Stimuli in Young Men,” Journal of Behavioral and Brain Science, Vol. 2, 2012, pp. 363-371. http://dx.doi.org/10.4236/jbbs.2012.23041

[12] A. Sanz-Martin, M. Hernández-González, M. A. Guevara, G. Santana and G. Gumá-Díaz, "Efecto del Alcohol en la Organización Eléctrica Cerebral Durante una Tarea de Memoria de Trabajo Visuoespacial y su Relación con el Ciclo Menstrual,” Revista de Neurología, Vol. 58, No. 3, 2014, pp. 103-112.

[13] F. M. Ferraro and S. W. Kiefer, "Behavioral Analysis of Male Rat Sexual Motivation and Performance Following Acute Ethanol Treatment,” Pharmacology, Biochemistry and Behavior, Vol. 78, No. 3, 2004, pp. 427-433. http://dx.doi.org/10.1016/j.pbb.2004.03.020

[14] K. Yoshimoto and W. J. McBride, "Regulation of Nucleus Accumbens Dopamine Release by the Dorsal Raphe Nucleus in the Rat,” Neurochemistry Research, Vol. 17, No. 5, 1992, pp. 401-407. http://dx.doi.org/10.1007/BF00969884

[15] K. Yoshimoto, K. Yayama, M. Ogata, A. Nishimura, T. Yoshida, S. Ueda, et al., "Possibility of 5-HT3 Receptor Involvement in Alcohol Dependence: A Microdialysis Study of Nucleus Accumbens Dopamine and Serotonin Release in Rats with Chronic Alcohol Consumption,” Alcoholism: Clinical and Experimental Research, Vol. 20, No. S9, 1996, pp. 311-319. http://dx.doi.org/10.1111/j.1530-0277.1996.tb01799.x

[16] M. S. Brodie, C. Pesold and S. B. Appel, "Ethanol Directly Excites Dopaminergic Ventral Tegmental Area Reward Neurons," Alcoholism: Clinical and Experimental Research, Vol. 23, No. 11, 1999, pp. 1848-1852. http://dx.doi.org/10.1111/j.1530-0277.1999.tb04082.x

[17] G. Di Chiara, “Alcohol and Dopamine,” Alcohol Health \& Research World, Vol. 21, No. 2, 1997, pp. 108-114.

[18] K. Kiianmaa, P. Hyytiä, H. H. Samson, J. A. Angel, L. Svensson, B. Söderpalm, et al., "New Neuronal Networks Involved in Ethanol Reinforcement," Alcoholism: Clinical and Experimental Research, Vol. 27, No. 2, 2003, pp. 209-219. http://dx.doi.org/10.1097/01.ALC.0000051020.55829.41

[19] Y. Tizabi, R. L. Copeland Jr., V. A. Louis and R. E. Taylor, "Effects of Combined Systemic Alcohol and Central Nicotine Administration into Ventral Tegmental Area on Dopamine Release in the Nucleus Accumbens," Alcoholism: Clinical and Experimental Research, Vol. 26, No. 3, 2002, pp. 304-399. http://dx.doi.org/10.1111/j.1530-0277.2002.tb02551.x

[20] F. Weiss and L. J. Porrino, "Behavioral Neurobiology of Alcohol Addiction: Recent Advances and Challenges," Journal of Neuroscience, Vol. 22, No. 9, 2002, pp. 33323337.

[21] L. Yavich and J. Tiihonen, "Ethanol Modulates Evoked Dopamine Release in Mouse Nucleus Accumbens: De- pendence on Social Stress and Dose,” European Journal of Pharmacology, Vol. 401, No. 3, 2000, pp. 365-373. http://dx.doi.org/10.1016/S0014-2999(00)00456-8

[22] C. J. Slawecki, "Altered EEG Responses to Ethanol in Adult Rats Exposed to Ethanol during Adolescence,” Alcoholism: Clinical and Experimental Research, Vol. 26, No. 2, 2002, pp. 246-254. http://dx.doi.org/10.1111/j.1530-0277.2002.tb02531.x

[23] M. Hernández-González, M. Martínez-Pelayo, M. Arteaga-Silva, H. Bonilla-Jaime and M. A. Guevara, "Ethanol Changes the Electroencephalographic Correlation of the Ventral Tegmental Area and Nucleus Accumbens, Components of the Mesoaccumbens System in Rats," Pharmacology Biochemistry and Behavior, Vol. 92, No. 1, 2008, pp. 124-130. http://dx.doi.org/10.1016/j.pbb.2008.10.020

[24] L. A. Mead and C. H. Vanderwolf, "Hippocampal Electrical Activity in the Female Rat: The Estrous Cycle, Copulation, Parturition, and Pup Retrieval," Behavioral Brain Research, Vol. 50, No. 1-2, 1992, pp. 105-113. http://dx.doi.org/10.1016/S0166-4328(05)80292-X

[25] M. Hernández-González, M. A. Guevara, M. Cervantes, G. Moralí and M. Corsi-Cabrera, "Characteristic Frequency Bands of the Cortico-Frontal EEG during the Sexual Interaction of the Male Rat as a Result of Factorial Analysis,” Journal of Physiology-Paris, Vol. 92, No. 1, 1998, pp. 43-50.

http://dx.doi.org/10.1016/S0928-4257(98)80022-3

[26] F. Lopes da Silva, "Neural Mechanisms Underlying Brain Waves: From Neural Membranes to Networks," Electroencephalography and Clinical Neurophysiology, Vol. 79, No. 2, 1991, pp. 81-93. http://dx.doi.org/10.1016/0013-4694(91)90044-5

[27] G. Paxinos and C. H. Watson, "The Rat Brain in Stereotaxic Coordinates,” Elsevier Academic Press, San Diego, 2005.

[28] M. A. Guevara, J. Ramos, M. Hernández-González, H. Madera and M. Corsi-Cabrera, "CAPTUSEN: A System for the Computerized Acquisition of EEG and Event-Related Potentials,” Mexican Journal of Psychology, Vol. 17, No. 1, 2000, pp. 77-88.

[29] A. Ågmo, A. Villalpando, Z. Picker and H. Fernández, "Lesions of the Medial Prefrontal Cortex and Sexual Behavior in the Male Rat,” Brain Research, Vol. 696, No. 1-2, 1995, pp. 177-186. http://dx.doi.org/10.1016/0006-8993(95)00852-H

[30] M. Hernández-González, C. A. Prieto-Beracoechea, M. Arteaga-Silva and M. A. Guevara, "Different Functionality of the Medial and Orbital Prefrontal Cortex during a Sexually Motivated Task in Rats,” Physiology \& Behavior, Vol. 90, No. 2-3, 2007, pp. 450-458. http://dx.doi.org/10.1016/j.physbeh.2006.10.006

[31] J. F. Lubar, T. J. Herrman, D. R. Moore and M. N. Shouse, "Effect of Septal and Frontal Ablations on Species Typical Behavior in the Rat," Journal of Comparative and Physiological Psychology, Vol. 83, No. 2, 1973, pp. 260-270. http://dx.doi.org/10.1037/h0034410

[32] A. Fernández-Guasti, I. Omana-Zapata, M. Luján and M. Condés-Lara, “Actions of Sciatic Nerve Ligature on Sex- 
ual Behavior of Sexually Experience and Inexperienced Male Rats: Effects of Frontal Pole Decortication,” Physiology and Behavior, Vol. 55, No. 3, 1994, pp. 577-581. http://dx.doi.org/10.1016/0031-9384(94)90119-8

[33] A. Sanz-Martin, M. A. Guevara, C. Amezcua, G. Santana and M. Hernández-González, "Effects of Red Wine on the Electrical Activity and Functional Coupling between Prefrontal-Parietal Cortices in Young Men,” Appetite, Vol. 57, No. 1, 2011, pp. 84-93.

http://dx.doi.org/10.1016/j.appet.2011.04.004

[34] E. Schwartz, P. Kielholz, L. Goldberg, U. Gilsdorf, M. Hofstetter, D. Ladewig, et al., "Alcohol-Induced Biphasic Background and Stimulus-Elicited EEG Changes in Rela- tion to Blood Alcohol Levels," International Journal of Clinical Pharmacology, Therapy and Toxicology, Vol. 19, No. 3, 1981, pp. 102-111.

[35] J. T. Coult, "Neural Correlates of Attention and Arousal from Electrophysiology, Functional Neuroimaging and Psychopharmacology," Progress in Neurobiology, Vol. 55, No. 4, 1998, pp. 343-361. http://dx.doi.org/10.1016/S0301-0082(98)00011-2

[36] V. N. Murthy and E. E. Fetz, "Coherent 25- to 35-Hz Oscillations in the Sensorimotor Cortex of Awake Behaving Monkeys," Proceedings of the National Academy of Sciences of the USA, Vol. 89, No. 12, 1992, pp. 56705674. http://dx.doi.org/10.1073/pnas.89.12.5670 